\title{
Equalization of MIMO-OFDM System under Time Varying Channel using ANFIS
}

\author{
Zeeshan A. Abbasi ${ }^{1 *}$ and Zainul Abdin Jaffery ${ }^{2}$ \\ 'University Polytechnic, Jamia Millia Islamia New Delhi - 110025, India; zaabbasi@jmi.ac.in \\ 2Department of Electrical Engineering, Jamia Millia Islamia, New Delhi - 110025, India; zjaffery@jmi.ac.in
}

\begin{abstract}
Background/Objective: High speed reliable communication for wireless internet is the main challenge for all communication engineers. Methods: Multiple Input Multiple Output (MIMO) Orthogonal Frequency Division Multiplexing (OFDM) is one possible solution, which promises high data rates. But MIMO-OFDM degrades the system performance in terms of Bit Error Rate (BER), due to Inter Symbol Interference (ISI), problem associated with multipath effect of the time varying wireless channels. To improve the performance of the MIMO-OFDM system a channel equalization technique is incorporated at the receiver end. Findings: The effectiveness of soft-computing approach in dealing the problem of non linear time varying channel is investigated in this paper by designing an equalizer based on Adaptive Neuro Fuzzy Inference System (ANFIS). The proposed technique is compared with the already tested Neural Network based equalizer. Result shows that the proposed equalizer gives better BER. Application/Improvements: The proposed equalizer can be implemented in wireless Local Area Networks (LAN) or mobile networks. The performance can be further improves with the increase signal length as evident by the graphs 14-16.
\end{abstract}

\section{Keywords: ANFIS, Equalization, MIMO, OFDM, Time Varying Channels}

\section{Introduction}

The need of all future wireless system is the design technologies which support high data rate to fulfil the need of multimedia based applications. MIMO-OFDM system is the emerging technology stands as promising choices for future high speed wireless communications ${ }^{\stackrel{1}{ } \text {. }}$

OFDM is a modulation technique which finds increasing number of uses in today's wireless communication scenario. It is being used in all emerging wireless technologies such as Digital Terrestrial TV, Digital Audio Broadcast (DAB), Wi-Fi upto 54 mbps in 802.11a standard, Wi-max and all higher generation cellular radio communication system². Channel Equalizer is an essential part of any communication system. It is used at the receiver end to combat the channel degradation effect specially Inter-Symbol Interference (ISI) and Co-Channel Interference (CCI). The OFDM signal can be easily equalize by 1 -tap equalizer for time invariant multipath channel ${ }^{3}$. For equalizing time varying channel different equalizer algorithms have been proposed for OFDM Communication in ${ }^{4.5}$. OFDM equalization using Neural

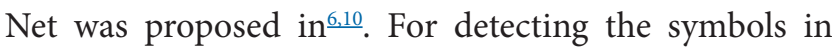
multiple antenna environment in MIMO systems in time varying channels adaptive equalizer algorithm in 6 may be employed. The DFE algorithm discussed in ${ }^{6}$ are useful for reducing ISI; however it is not suitable for removing CCI generated by multiple channels.

\section{MIMO-OFDM System Model}

OFDM is a modulation scheme that splits the total available spectrum into a number of sub-channels which are orthogonal to each other. For discrete time signal the orthogonality is:

$\sum_{k=0}^{N-1} \cos \left(\frac{2 \pi k n}{N}\right) x \cos \left(\frac{2 \pi k m}{N}\right)=0 \quad(\mathrm{n} \neq \mathrm{m})$

${ }^{*}$ Author for correspondence 
Where $\mathrm{N}=$ Period of $\mathrm{k}$ samples.

In multicarrier modulation system, symbols are assigned to different orthogonal carriers (called subcarrier) and all these carriers are combined together by IFFT technique before transmission. This combined signal is called OFDM baseband. Figure 1 shows a typical case in which three symbols are assigned to a carrier²
MIMO uses the multiple antennas at both transmitting and receiving end. It adds many additional channels which are parallel to each other in spatial domain. These parallel channels established high data rates in the same bandwidth ${ }^{7}$. MIMO-OFDM system can be used for all applications which require high data rates ${ }^{\underline{8}}$. MIMO-

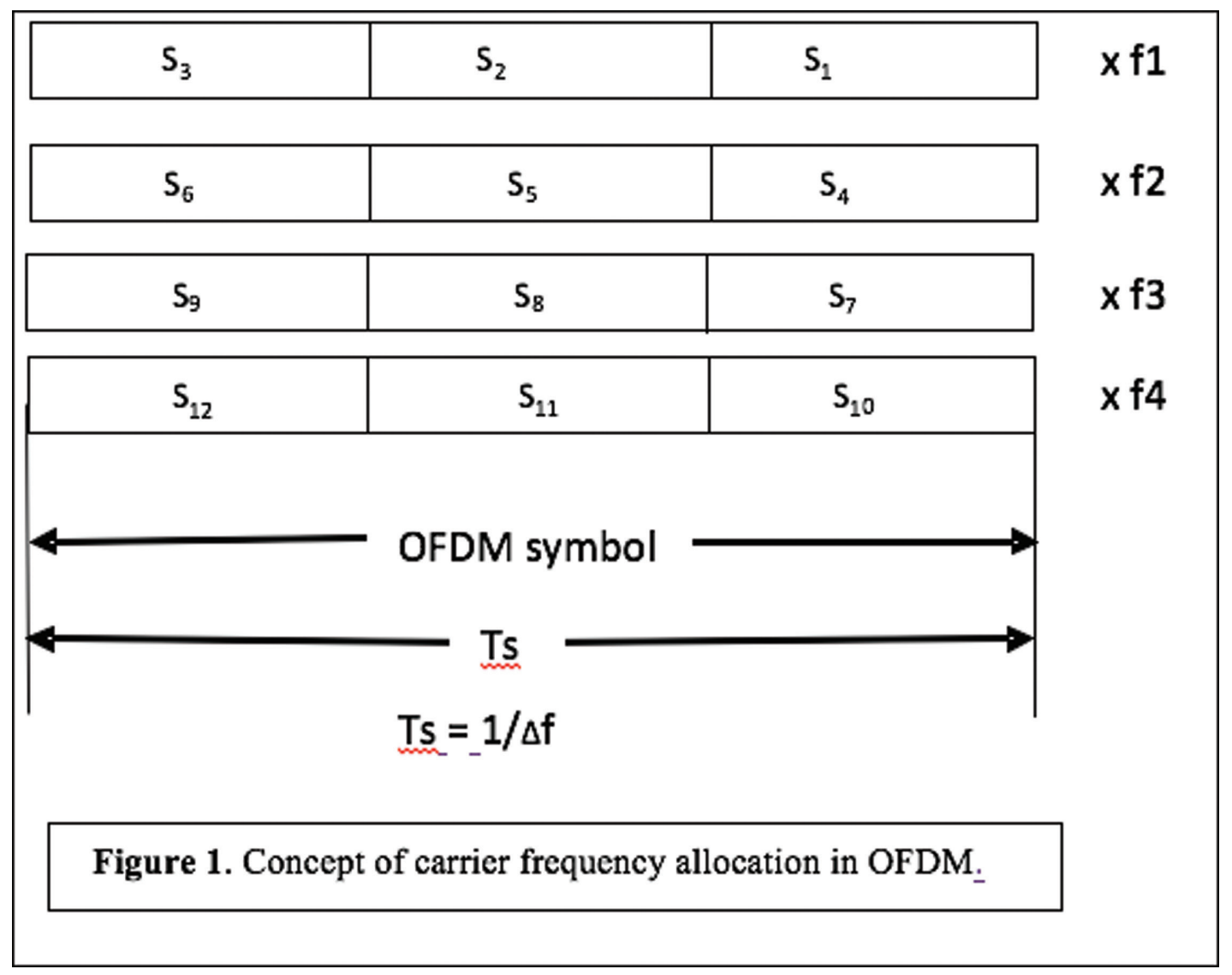

Figure 1. Concept of carrier frequency allocation in OFDM.

OFDM system with two transmitting and receiving antennas are shown in Figure 2.

According to Shannon, the channel capacity for SISO is given by the relation:

$$
C=W \log _{2}(1+S N R)
$$

Where $\mathrm{W}$ is the channel bandwidth.

For MIMO with $\mathrm{M}$ transmitting antennas and $\mathrm{N}$ receiving antennas the equation 1 can be written as:

$$
C=W \log _{2}(1+M N \cdot S N R)
$$

\subsection{MIMO-OFDM Transmitter Model}

Figure 3 shows the block diagram of MIMO-OFDM system with two transmitting antennas. In digital communication the information to be transmitted are expressed in the form of bits. The input serial bit stream is read into the symbols, collection of various sizes of bits ${ }^{5}$. These symbols are used to generate OFDM data by taking symbols in the spectral apace using M-PSK, M-QAM technique. In the proposed method 16-QAM is used as it gives the best bandwidth efficiency ${ }^{\underline{6}}$. In multi transmitting antenna type Space Time Block Code (STBC) is used so that individual antenna can transmit L successive 


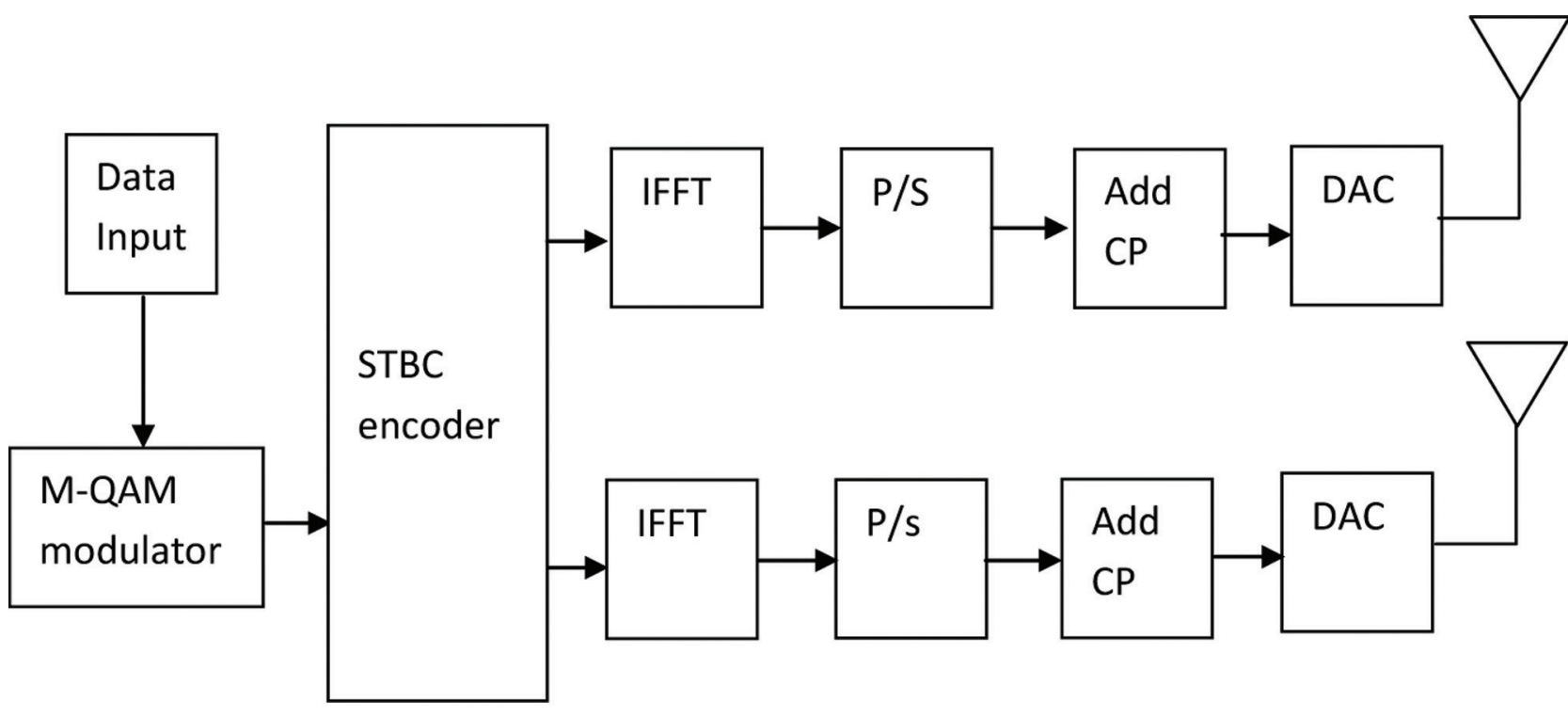

Figure 3. MIMO-OFDM Transmitter with Two Transmitting antennas

symbols in permutation manner at each signalling interval. In proposed system two successive symbols are stored say $S_{1}$ and $S_{2}$. At the first signalling interval first antenna will transmit $S_{1}$ whereas second antenna will transmit $S_{2}$. At second signalling interval first antenna will transmit $-S_{2}^{*}$ and second antenna 2 will transmit $S_{2}^{*}$. This can be expressed in matrix form as:

$$
\left[\begin{array}{cc}
S_{1} & -S_{2}^{*} \\
S_{2} & \mathrm{~S}_{1}^{*}
\end{array}\right]
$$

The generate spectra is converted to time domain using IFFT as it is more cost effective to implement as compare to Inverse Discrete Fourier Transform (IDFT) $\underline{\underline{z}}$. ISI, a common problem in high data rate communication, can be reduced by adding a guard interval. The most effective guard period to use is the cyclic prefix $x^{2}$. Hence before $\mathrm{RF}$ conversion we add cyclic prefix to compensate for ISI effect. As the output of the IFFT stage is in the form of discrete samples, digital to analog conversion is required before the RF conversion stage.

\subsection{MIMO-OFDM Receiver Model}

Figure 4 shows the MIMO-OFDM Receiver model. The $\mathrm{RF}$ down conversion process is the reverse process performed at the receiver front end. At this stage, the cyclic prefix is removed and serial to parallel conversion is done. The FFT of each OFDM symbol is performed to find the original transmitted spectrum. In the detection part we have two options to inverse the distortion due to channel. First we can do channel estimation followed by channel equalization as discussed in $\frac{6.8}{}$ or we can do channel equalization directly based on certain criterion such as Minimum Mean Square Error (MMSE) or Bit Error Rate (BER). In the proposed system only channel equalization is done and then finally the demodulation is carried out to recover the original bit stream.

\section{Channel Equalization}

Equalization is the process of recovery of a signal that is distorted by the non linearity of the channel. Equalizer is incorporated at the receiver such that its transfer function is exactly reciprocal to the channel transfer function? 2 . 


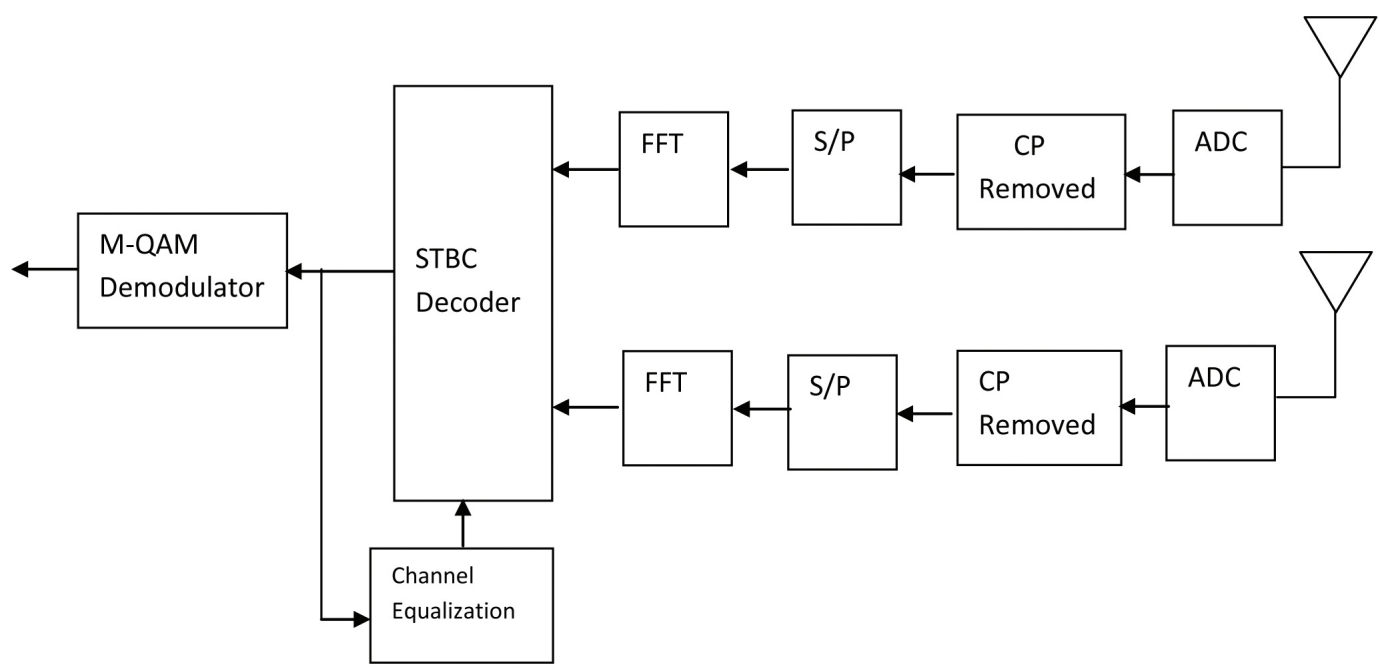

Figure 4. MIMO-OFDM Receiver with Two Receiving

The concept of equalization can be represented mathematically using the following equation:

$$
r(n)=h(n) * s(n)+w(n)
$$

Where $r(n)$ is the output of the channel, $h(n)$ is the channel impulse response, $s(\mathrm{n})$ is the input signal to the equalizer and $\mathrm{w}(\mathrm{n})$ is the AWGN noise.

If noise is removed using a filter then the original signal can be detected with the removal of the channel effects and phase ambiguities. $S^{\prime}(n)=h^{-1}(n) * r(n)$

From Equation 4 it is clear that the equalization problem is relatively simple if the channel response is known and time invariant. But in most practical cases, the channel response is unknown, time-varying and non-linear thus an adaptive equalizer is required. Adaptive equalizer is a technique that automatically adapts to the time varying properties of the communication channel.

\subsection{Adaptive Neuro Fuzzy Inference System for Channel Equalization}

Figure 5 gives the general Structure for ANFIS. It consists of nodes and directional links which connect the various nodes?

$A_{1}, A_{2}, B_{1}, B_{2}$ are the nodes of layer 1 which is the input layer. Each node is a fuzzy set.The number of nodes are equal to the sum of external inputs. The output of layer 1 can be calculated as:

$$
o_{i}^{1}=\mu_{A i}(x)
$$

Where $\mathrm{x}$ is the input to node 1 and $\mathrm{Ai}$ is the linguistic label (small, medium, large). $o_{i}^{1}$ is the membership function of Ai which species the degree to which the input satisfies the quantifier Ai. $\mu_{A i}(x)$ is taken as bell shaped but any other membership function such as trapezoidal or triangular can also be considered. In this layer the parameters are referred as premise parameters.

Layer 2 is called the fuzzification layer. In this layer each node corresponds to one linguistic term. The output of this layer is the product of two incoming signals.

$$
w_{i}=\mu_{A i}(x) \times \mu_{B i}(y), i=1,2
$$

Layer 3 is labelled as $\mathrm{N}$ and it is a fuzzy rule layer. The number of nodes in this layer is equal to the number of rules. Output of this layer is the ratio of the $i^{\text {th }}$ rule's firing strength to the sum of all rules firing strengths:

$$
\overline{w_{i}}=\frac{w_{i}}{w_{1}+w_{2}}
$$

Layer 4 is the output membership layer. The output membership neuron combines all its inputs by using either fuzzy union or fuzzy intersection operation. For combining one can use either probabilistic OR or probabilistic AND.

$$
O_{i}^{4}=w_{i} f_{i}=w_{i}\left(p_{i} x+q_{i} y+r_{i}\right)
$$




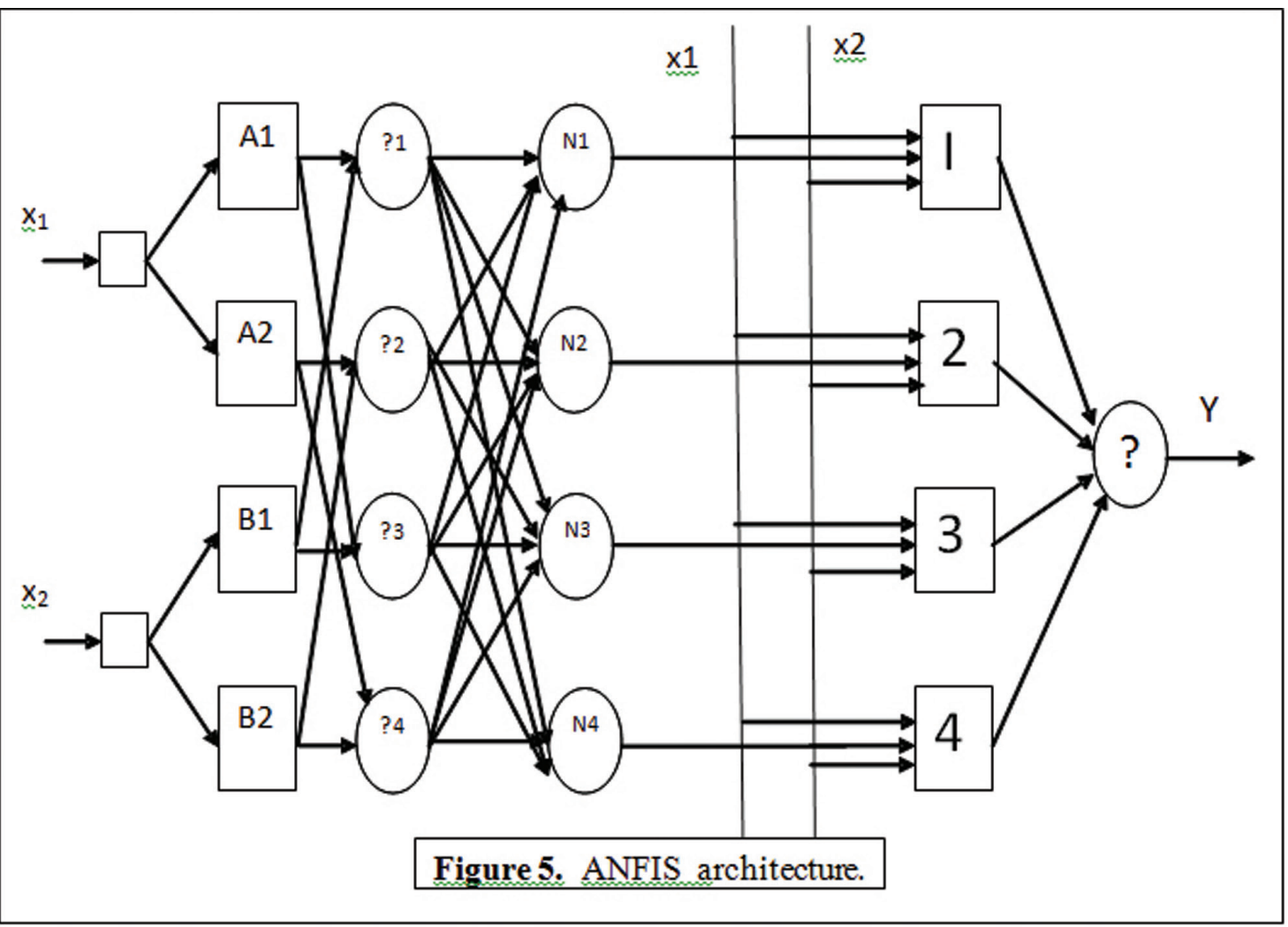

Where $\mathrm{w}_{\mathrm{i}}$ is the output of layer 3 and $\mathrm{p}, \mathrm{q}, \mathrm{r}$ are the consequent parameters.

Layer 5 generates the overall output of the neurofuzzy system. It is the defuzzification layer.

$$
o_{i}^{5}=\sum w_{i} f_{i}
$$

\subsection{ANFIS Equalizer}

To solve the problem of fast varying non-linear channel equalization, the solution other then the best available Neural Network based Channel Equalizer, is ANFIS based equalizer ${ }^{11}$. Structure for ANFIS used for channel equalization is given in Figure 6. Fuzzy based equalizers based on Fuzzy IF-THEN rules and incorporate numerical and linguistic information improves the adaptation speed and Bit Error Rate (BER), but its system time response slows down. ANFIS equalizer overcome this drawback and retains its advantage. Here to design ANFIS Equalizer first order Takagi and Sugeno (TSK) FIS model is chosen. We take two inputs, 7 membership functions and 49 rules. The standard deviation of the output of the channel is used as the antecedent parameters whereas the scaling factor is used as the consequent parameters. The membership function is chosen as the Gaussian. The rules are formulated and store in the fuzzy rule based. The output of the equalizer is given to the limiter to clip the output to the limiting value of +1 and -1 . The equivalent structure for equalizer is shown in the Figures 7 and 8 .

If we assume $\sigma$ as the standard deviation $\rho$ as the scaling factor then some of the rules store in the rule based may be summarized as:

If $\sigma$ of the received signal is very low and $\sigma$ of the collective interference is very low and $\sigma$ of the noise is very low then output $y=\rho_{1} x$.

If $\sigma$ of the received signal is low and $\sigma$ of the collective interference is low and $\sigma$ of the noise is low then output $y=\rho_{2} x$.

If $\sigma$ of the received signal is low and $\sigma$ of the collective interference is very low and $\sigma$ of the noise is medium then output $y=\rho 3 x$.

If $\sigma$ of the received signal is high and $\sigma$ of the collective interference is medium and $\sigma$ of the noise is low then output $y=\rho_{4} x$. 


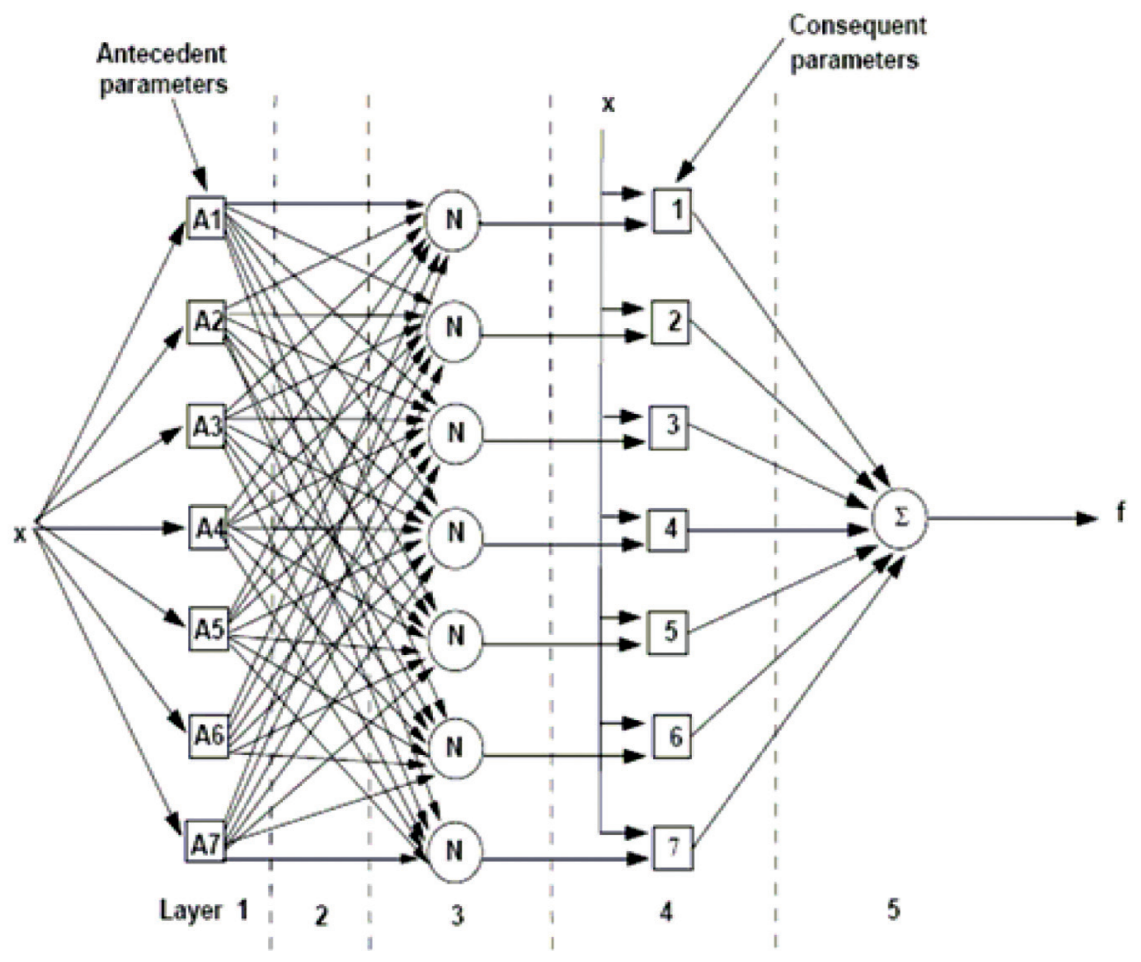

Figure 6 ANFIS Channel Equalizer

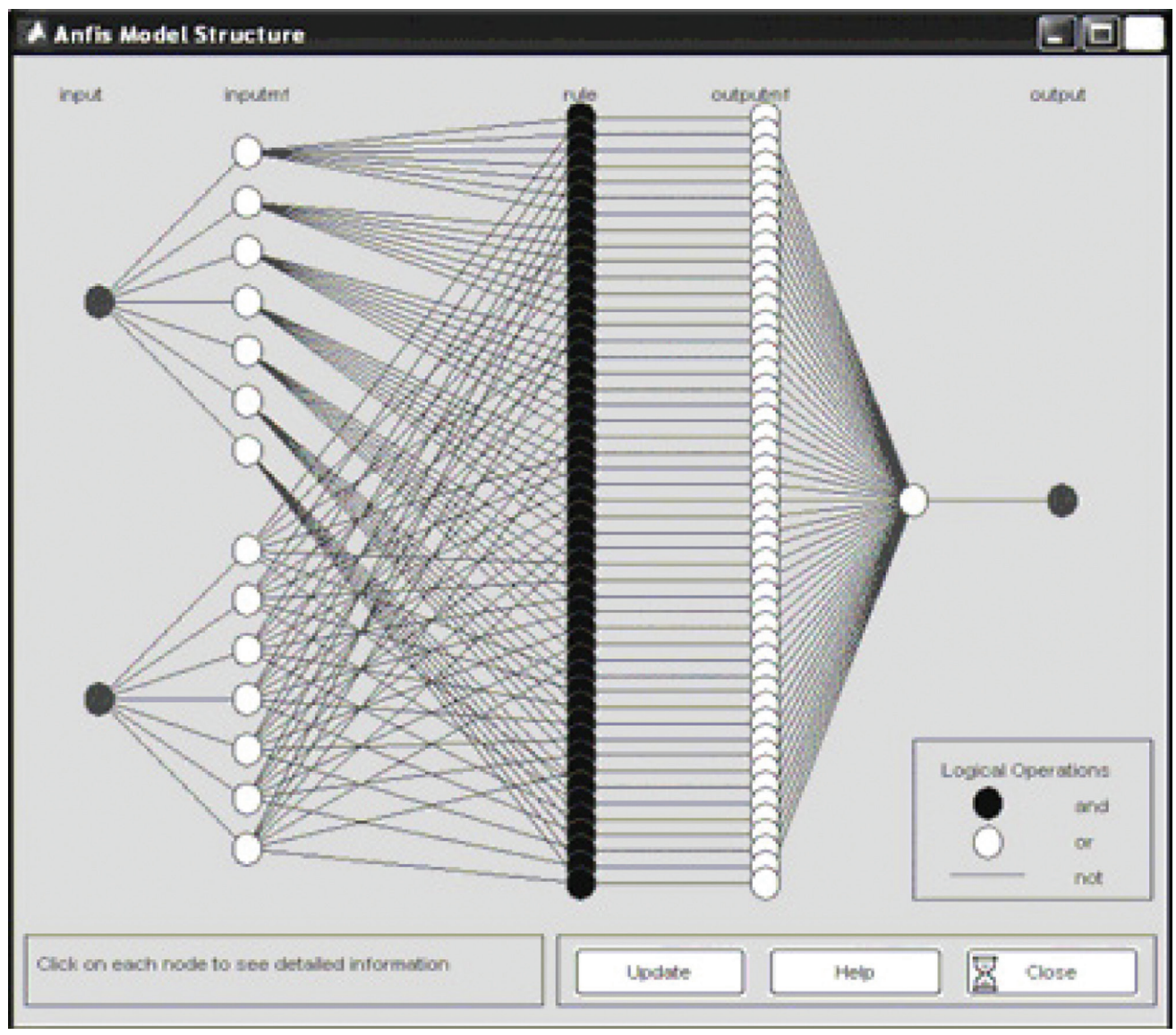

Figure 7 ANFIS Model Structure 


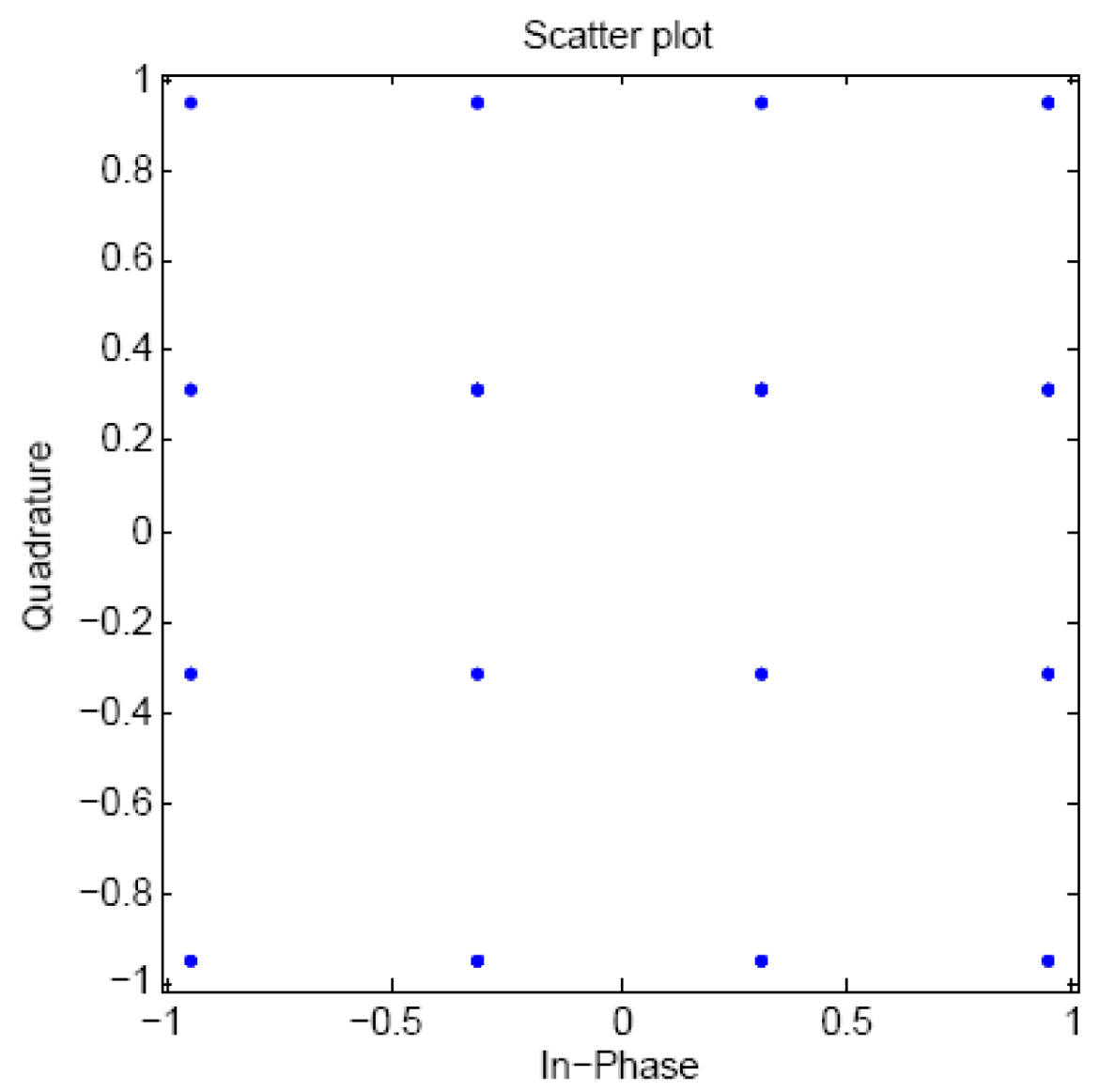

Figure 8. 16-QAM constellation.

\section{Simulation and Results}

In this section the simulation parameters and the results generated will be discussed. The following parameters are chosen for OFDM transmission.

Signal Length $=500$; Channel Length $=20$; Channel Type $=$ Rayleigh Channel $;$ Number of subcarriers $=64$; Cyclic prefix length $=16$; No of antennas $=2$; The generated data are modulated using 16 QAM will look like as shown in Figure 8, before adding the cyclic prefix this data is taken as the training data. The data after up-conversion passes through the channel and get distorted by the multipath effect of the Rayleigh channel and AWGN noise. The distorted data will look like as shown in Figure 9. This data is used as the target data. With the help of these data the Neural Network based and ANFIS based equalizers are tuned. After equalization the received data will look like as in Figure 10. Now these received data are demodulated and with these we calculate the BER for both the equalizers and the graphs are plotted for comparison. Finally the ANN and ANFIS are trained for 20 epochs and its performance is measured by mean square error as shown in Figure 11. It is clear from the graph that the MMSE for ANFIS equalizer are good from the beginning at the low values of SNR and become equal to NN Equalizer for higher values of SNR.

The results are very promising for BER as can be seen from Figure 12 onwards. The ANFIS gives better BER as compare to ANN based equalizer. The BER of ANFIS based equalizer is very close to the analytical value and it further improves and become closer to its analytical value as the signal lengths are increases whereas the NN based equalizer shows no improvements it is easily evident from Figure 13 to 16. 


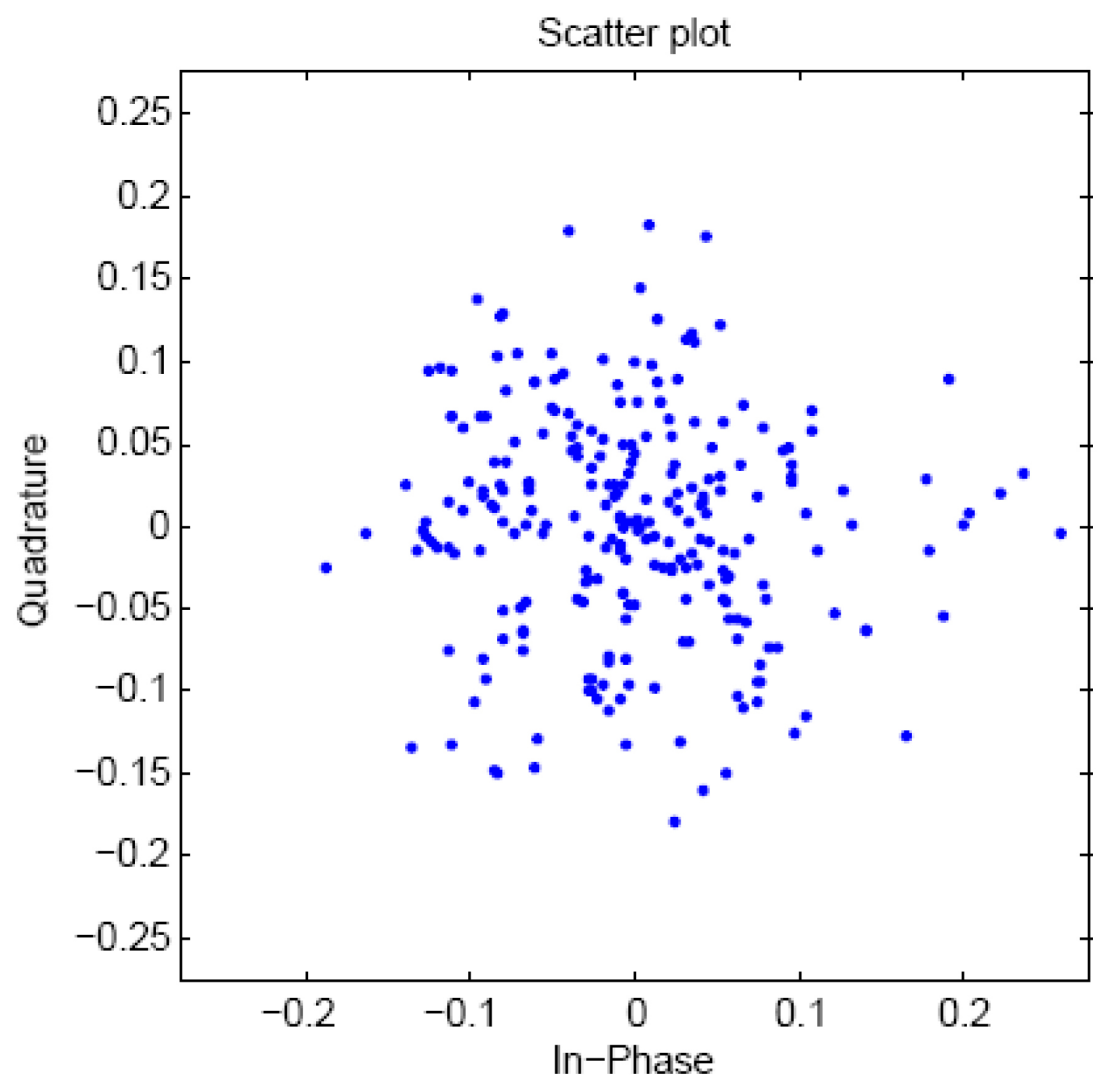

Figure 9. Before equalization.

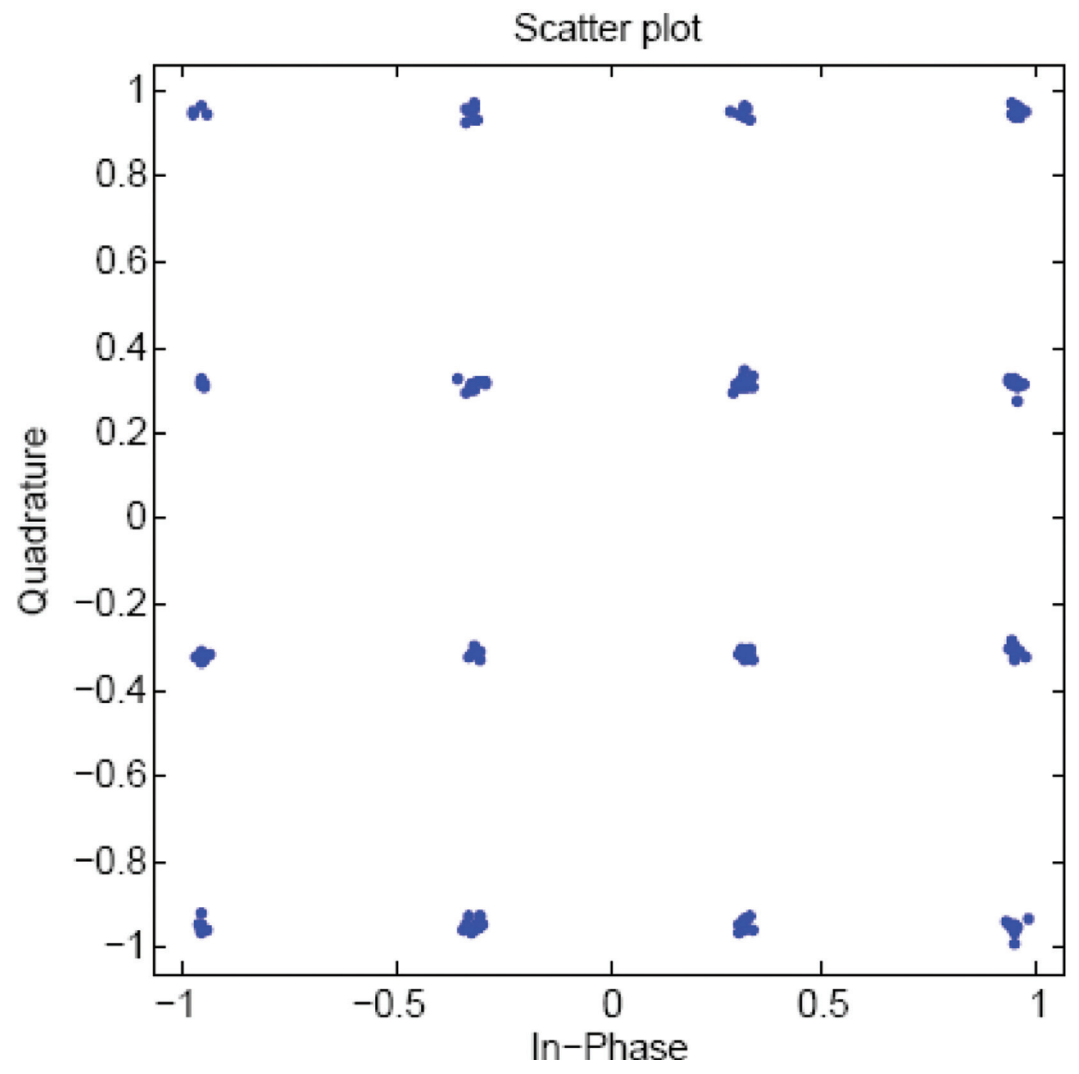

Figure 10. After equalization diagram. 


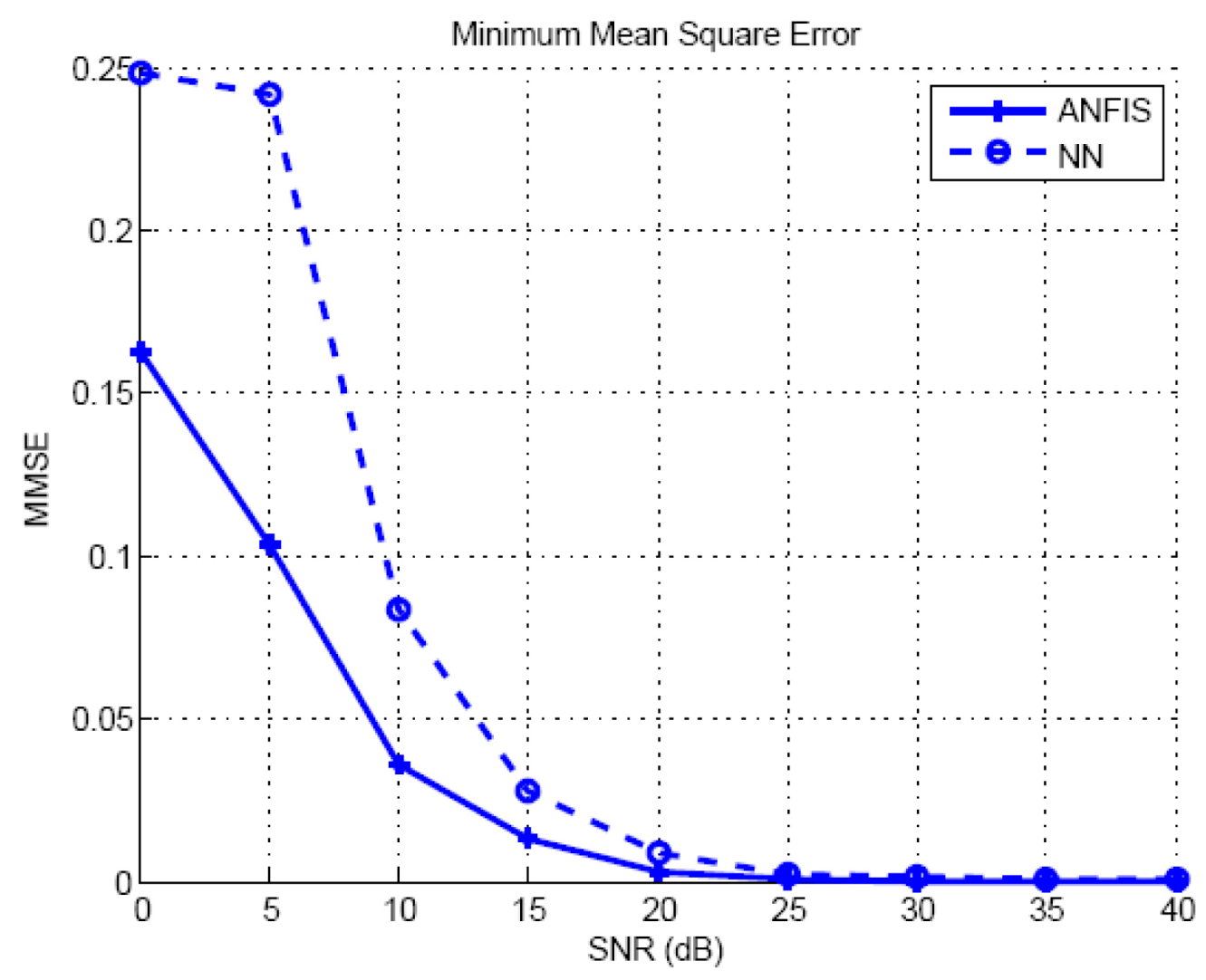

Figure 11. SNR vs. MMSE.

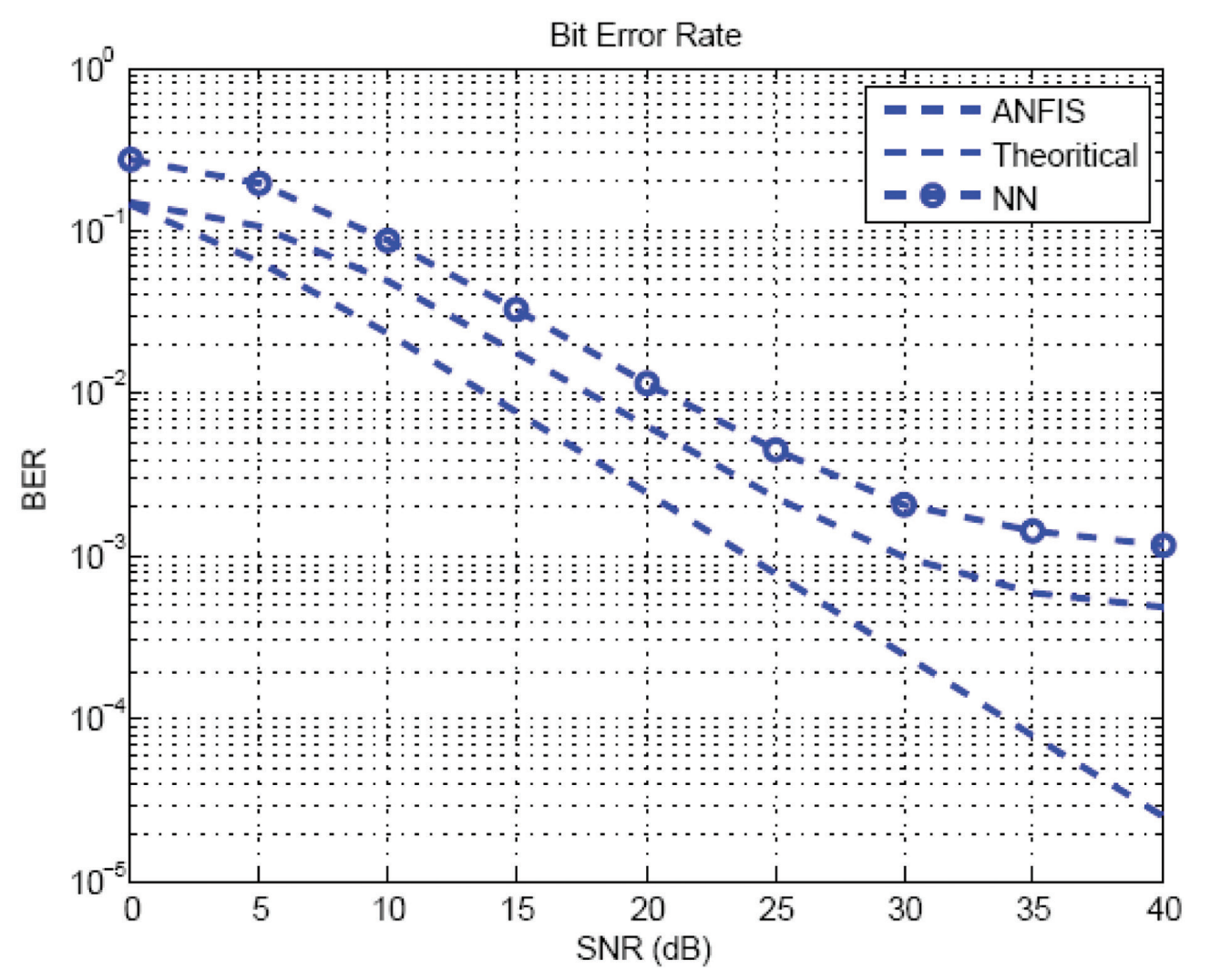

Figure 12. SNR vs BER. 


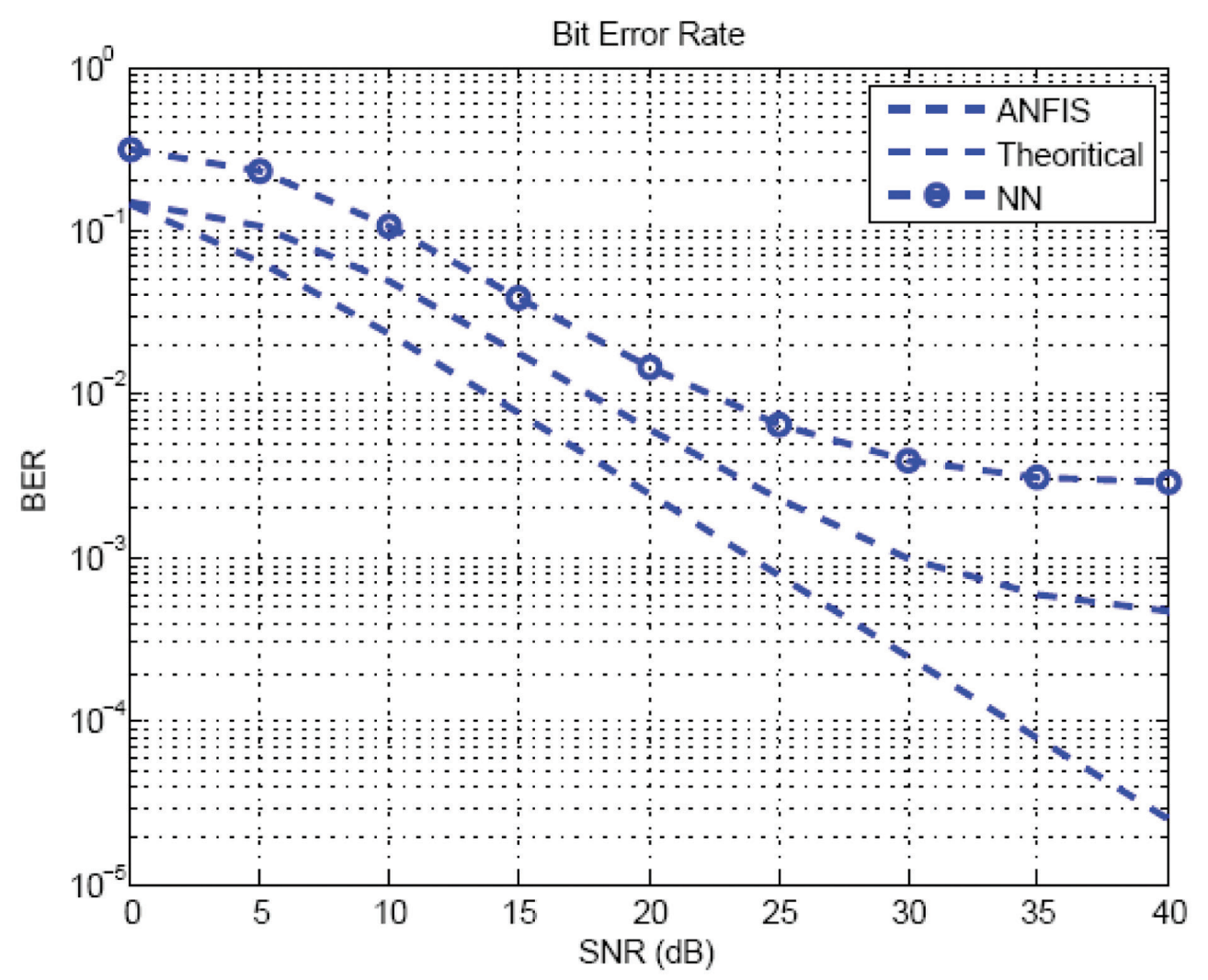

Figure 13. Signal length double/

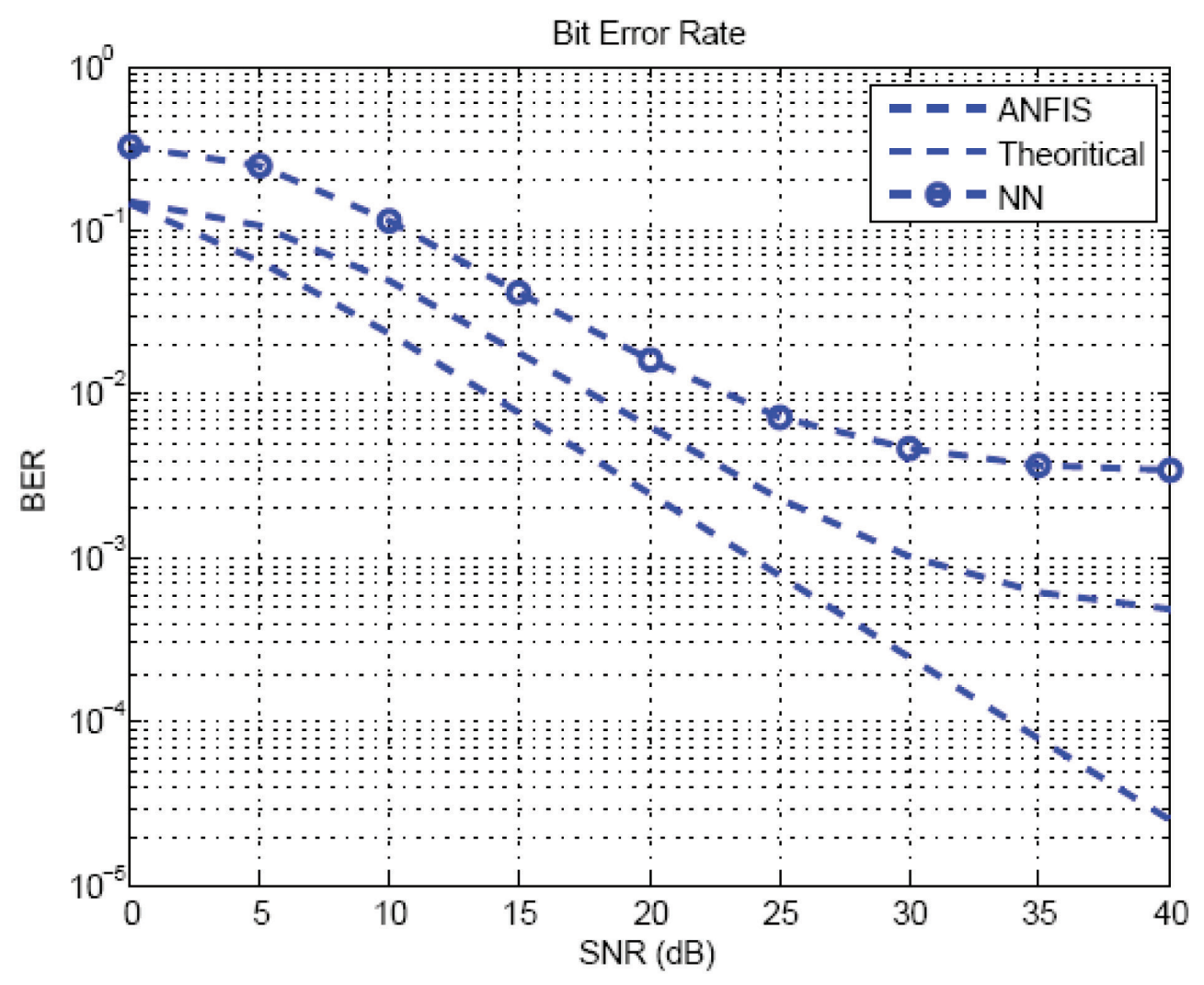

Figure 14. Signal length triple. 


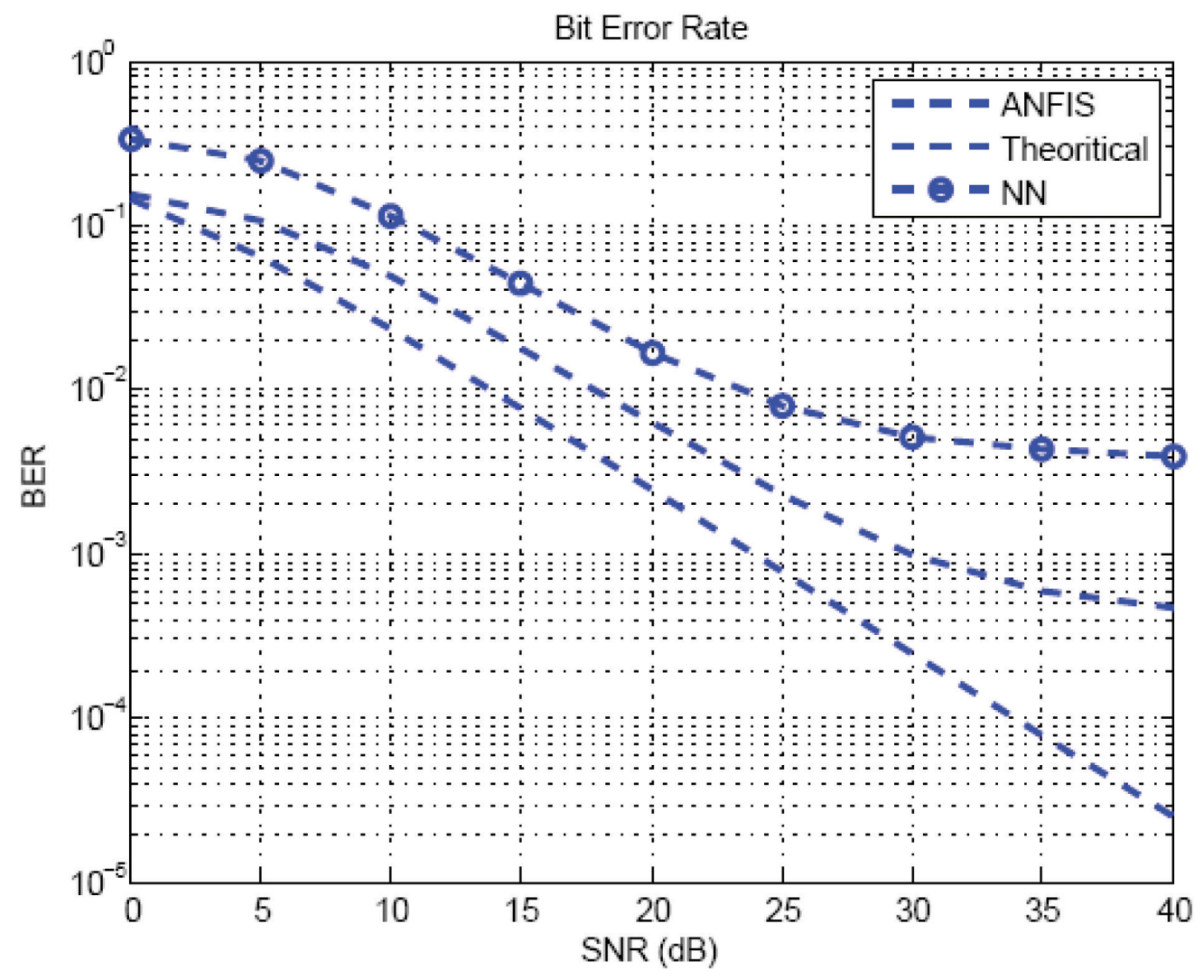

Figure 15. Signal length four times.

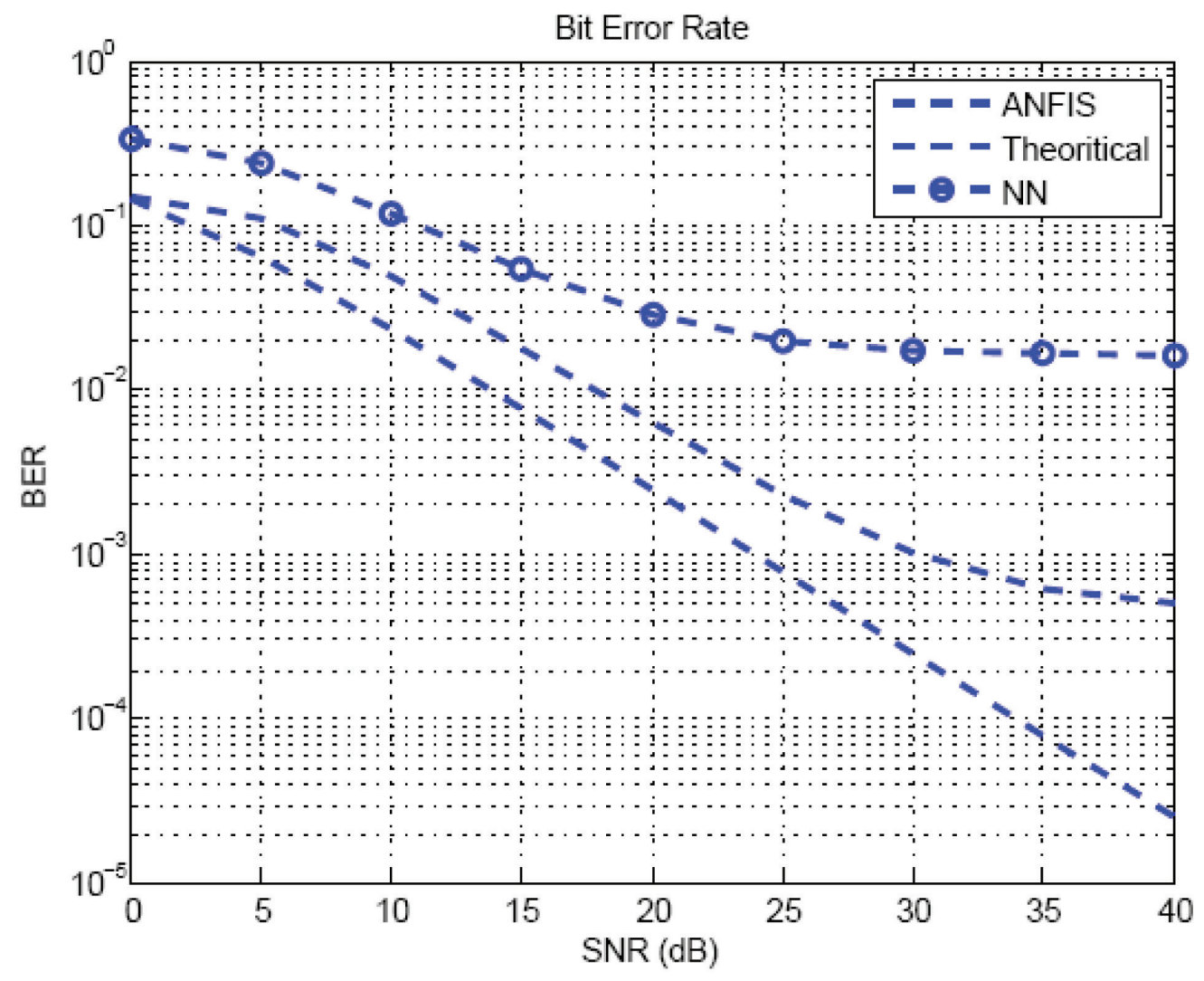

Figure 16. Signal length ten times. 


\section{Conclusion}

In this paper the results of two channel equalizers, NN based and ANFIS based are compared on MIMO-OFDM system over Rayleigh fading channel. The application of Neural Network in channel estimation has already been tested in ${ }^{12}$. The same idea is applied in channel equalization and the results are compared with the propose ANFIS based equalizer. ANFIS based equalizer performs better as compared to the NN based and the performance can be further improved as the signal length is increased.

\section{References}

1. Foschini GJ, Gans MJ. On limits of wireless communications in a fading environment when using multiple antennas. Wireless Pers Communication. 1998 Mar; 6(3):311-35. Crossref.

2. Dalal U. Wireless Communication and Networks. 1st ed. Oxford university Press. 2015.

3. Wang Z, Giannakis GB. Wireless multicarrier communications: Where Fourier Meets Shannon. IEEE Signal Processing Magazine. 2000 May; 17(3):29-48. Crossref.

4. Jeon WG, Chang KH, Cho YS. An equalization technique for OFDM systems in output (MIMO) channel. Proceedings of IEEE ICC; Helsiniki, Finland. 2002 Jan. p. 59-63.
5. Wolniansky PW. V-Blast: An architecture for realizing very high data rates over the rich scattering wireless channels. Proceedings of IEEE Symposium on Signal System and Electronics; 1998. p. 295-300. Crossref.

6. Zelst AV, Schenk TCW. Implementation of MIMO-OFDM based wireless LAN System. IEEE Trans Signal Processing. 2004 Feb; 52(2):483-94. Crossref.

7. Pandharipande A. The principles of OFDM. IEEE Potentials. 2002; 21(2):16-19.

8. Sanjana T, Suma MN. Comparison of channel estimation and equalization techniques for OFDM Systems. Circuits and System: An International Journal (CSIJ). 2014 Jan; 1(1):1-10.

9. Acosta G. OFDM simulation using MATLAB. 2000 Aug. p. $1-20$.

10. Tao J, Zheng YR, Yang WB. Channel equalization for single carrier MIMO underwater acoustic communications. EURASIP Journal on Advances in Signal Processing. 2010 Jun. Crossref.

11. Raveendranathan KC, Harisankar M, Kaimal MR. A new class of ANFIS based channel equalizer for mobile communication systems. IJSSST. 2010; 11(1):1-7

12. Sharma K, Varshney S. Artificial Neural Network channel estimation for OFDM system. IJECSE. 2012 Aug; 1(3):1686-91. 